Address for Correspondence: Dr. Patrick G. Northup, MD,

Medical Director of Liver Transplantation

Section Chief, Hepatology

Associate Professor of Medicine

University of Virginia

Charlottesville, VA 22908-0708, USA

Email: northup@virginia.edu

\begin{tabular}{|l|}
\hline Access this article online \\
\hline Website: \\
www.intern-med.com \\
\hline DOI: \\
10.2478/jtim-2018-0001 \\
\hline Quick Response Code: \\
\hline
\end{tabular}

\title{
Timing of anticoagulation for portal vein thrombosis in liver cirrhosis: A US hepatologist's perspective
}

\author{
Patrick G. Northup, Jessica P. E. Davis \\ Center for the Study of Coagulation in Liver Disease; Division of Gastroenterology and Hepatology, \\ University of Virginia,Charlottesville, Virginia, USA
}

\section{INTRODUCTION}

Portal vein thrombosis (PVT) is a common event in patients with cirrhosis. Routine evaluations of the portal vascular system are performed semiannually in many patients with cirrhosis as they undergo hepatocellular carcinoma screening with Doppler ultrasound. Because the sensitivity and specificity of ultrasound, contrast enhanced computed tomography (CT) and magnetic resonance imaging (MRI) vary for PVT detection, incidence and prevalence estimates for PVT in the cirrhosis population vary widely based on the imaging modality used for diagnosis. Epidemiologic and cross sectional studies estimate the true prevalence of nonmalignant main trunk PVT to be between 2 and 8 percent in patients with various stages of cirrhosis. ${ }^{[1-3]}$ Because there are significant differences in the physiologic consequences of small intrahepatic PVT versus large occlusive clots in the main portal vein and/or the mesenteric system, several classification systems have been developed to describe the extent of clot, ${ }^{[3,4]}$ but there is no established consensus on the clot classification that directly correlates to outcome. Cross sectional and epidemiologic data support the intuitive argument that PVTs which are completely occlusive and have greater extension (i.e., mesenteric involvement) are associated with higher morbidity, increased technical difficulty during liver transplantation, and increased mortality after liver transplantation..$^{[5,6]}$ More intriguingly, a highly criticized but randomized controlled trial in patients at high risk for PVT using enoxaparin in prevention of PVT over the course of a year showed that low molecular weight heparin (LMWH) was not only highly effective at preventing the formation of PVT in the treatment arm but also resulted in reduced rates of portal hypertension decompensation and improved all cause survival in that group. ${ }^{[7]}$ While definitive prospective data on therapeutic intervention in PVT are lacking, it is clear that modern day hepatologists need to have an assessment, treatment, and monitoring strategy for PVT, especially in liver transplant candidates.

\section{DIAGNOSTIC STRATEGY AND CLASSIFICATION OF EXTENT OF PVT}

In cirrhosis patients, the literature suggests that more than $70 \%$ of partial or nonocclusive PVTs spontaneously resolve when initially discovered on Doppler ultrasonography. ${ }^{[8]}$ This high rate of spontaneous resolution is likely due to the inherent weaknesses in Doppler techniques. Unlike the deep veins in the extremities, the deep veins of the abdomen are not externally compressible; thus, differentiating true thrombosis versus sluggish flow is difficult. For this reason, before therapeutic decisions can be made regarding PVT, the diagnosis must be verified by contrast enhanced imaging such as CT or MRI. Importantly, malignant tumor thrombus, a common finding with hepatocellular carcinoma (HCC), does not respond to antihemostatic medications and HCC with tumor thrombus should be diagnosed and treated differently than bland, nonmalignant PVT. Once the diagnosis of nonmalignant PVT is confirmed, it is reasonable 
to decide on the treatment strategies and urgency based on the extent of thrombosis and presentation.

Extensive, occlusive, acute thrombosis of the combined portal and mesenteric system is rare but can be devastating. This syndrome often presents with signs and symptoms of small bowel ischemia and is frequently life threatening. Patients presenting with this disorder often have coexisting undiagnosed hematologic malignancy or thrombophilia. In the rare patient that presents with this acute, severe syndrome, decision making must be quick as mortality is high and emergency intervention to restore perfusion to the gut is paramount. This often involves thrombolysis, surgery, and interventional vascular procedures. This presentation is uncommon in a cirrhosis patient and frequently the care of these patients is overseen by surgical and hematologyoncology specialists.

The much more common presentation in the cirrhosis patient is the incidental presentation of PVT discovered during screening for HCC. The patient in this scenario typically has clinically significant portal hypertension and may either be asymptomatic or have symptoms of a recent increase in portal hypertension including increased ascites, acute bleeding from esophageal varices, or less commonly a flare of hepatic encephalopathy. Once the diagnosis is verified by cross sectional imaging, and malignancy - especially HCC - is ruled out as a source of venous thrombosis, attention should be turned to the control of the patient's portal hypertension complications. Increased diuretics may be required and therapy for high-risk varices may be needed. While there are some concerns that nonselective beta-blockers may decrease portal pressures and contribute to sluggish flow in the portal system thereby raising the risk for continued PVT, clinical data is sparse on this topic so these should be given when indicated according to the current practice guidelines. ${ }^{[9]}$ If there are multiple thromboses at presentation or if the patient has a first degree relative with thrombophilic syndromes or unusual clotting histories, it is reasonable to screen the patient for additional thrombophilic disorders, but this practice is low yield and is not generally recommended in a cirrhosis patient with typical PVT. ${ }^{[10]}$

\section{PATIENT SELECTION}

Once portal hypertension complications, especially esophageal varices are controlled, the decision for therapeutic intervention in incidentally discovered PVT should be handled on a case-by-case basis. If the patient has extreme comorbidities, very advanced liver disease, or absolute or relative contraindications to therapeutic interventions, then specific PVT therapy is unlikely to alter the disease course or symptomatology and no additional therapy other than portal hypertension control is indicated. Similarly, small intrahepatic sub-segmental PVT should raise the concern for early HCC but generally have no physiological risk other than the possibility of propagation and generally do not benefit from therapy. The clinician will often follow these small thromboses over time by repeat imaging at 3-6 month intervals to assess for propagation or definitive evidence of early HCC. Finally, patients presenting with evidence of fully collateralized chronic PVT, or cavernoma, with complete obliteration of the native portal vein are very unlikely to gain recanalization of physiologic portal flow and do not benefit from antihemostatic therapy. ${ }^{[1]}$

Special attention should be devoted to the liver transplant candidates or those patients presenting with complete occlusive main trunk PVT because these patients are most likely to benefit from therapeutic interventions. In the liver transplant candidates, the establishment of a patent main portal vein generally allows for endto-end anastomosis of the donor to recipient portal veins. This reconstruction of physiologic flow avoids venous jump grafts that might predispose to postoperative complications and have been linked to decreased survival after orthotopic liver transplant. ${ }^{[12]}$ While definitive interventional data with controls have not been published, it is reasonable to attempt to preserve or restore the main portal vein flow prior to transplantation if possible, to avoid non-physiologic reconstructions during transplant. Similarly, although there are cross sectional data suggesting that PVT may not be the cause of worsening hepatic function per se, ${ }^{[8]}$ theoretically, in the non-transplant patient with occlusive main PVT obstruction, improving flow in the main portal vein should lead to easier control of portal hypertension and might lead to an improvement in hepatic perfusion and prevention of cavernoma formation. These two patients, the transplant candidate and the patient with completely occlusive symptomatic main PVT thrombosis without cavernoma, are the cases that should be considered for directed therapy related to the PVT, as they have the most theoretical gain from treatment.

\section{THERAPEUTIC OPTIONS}

The choice of therapy for PVT in cirrhosis patients is not established and there have not been head to head controlled trials in this area. The evidence for recommendations on this topic is sparse and tends to be extrapolated from older data related to hepatic vein thrombosis and posttransplant or post-operative PVT. ${ }^{[10]}$ These extrapolations are tenuous at best as these disorders predominantly occur in non-cirrhosis patients and involve distinctly different presentations and overall management strategies. The ideal strategy for the treatment of PVT in patients with cirrhosis 
should involve minimal risk of complications related to the therapy, be simple and convenient to follow and administer, have a reasonable success rate in reestablishing physiologic flow in the portal system, and be easily terminated when the risk for PVT is gone (i.e., transplantation). This ideal therapy does not currently exist, and clinical trials have not been performed in this arena to identify the best therapy. The two most cited options include long term medical therapeutic anticoagulation and transjugular intrahepatic portosystemic shunt (TIPS) with mechanical or intraprocedural thrombolysis.

Therapeutic medical anticoagulation is an attractive option since it is widely available, does not require specific procedural techniques, can be started and stopped as needed, has low rates of specific complications, and does not permanently alter anatomy. There are numerous available antihemostatic medications across the world that have been extensively studied in cardiovascular disease and venous thromboembolism and medical practitioners are facile with these agents in the non-cirrhosis population. With an increased understanding of the hemostasis system in patients with chronic liver disease in recent years and knowledge that variceal bleeding is primarily driven by uncontrolled portal hypertension (as opposed to coagulopathy), ${ }^{[13]}$ practitioner comfort with the application of antihemostatic agents in cirrhosis patients has increased. These agents are now used more frequently in cirrhosis patients for a variety of indications. More than 18 controlled studies or case series have been reported in the treatment of PVT with medical agents including the vitamin $\mathrm{K}$ antagonists (VKA), low molecular weight heparins, and more recently, the direct acting oral anticoagulants (DOAC) inhibiting factor $\mathrm{X}$ or thrombin. ${ }^{[11]}$ One systematic analysis showed the efficacy rate of recanalization of PVT to be between 37-93\% depending on the extent of clot and time length between clot identification and treatment initiation. ${ }^{[1]}$ There have not been head-to-head trials comparing efficacy of any of these agents to placebo or to another agent in the class,so no data driven recommendations can be made regarding the choice of agent.

From a pharmacologic standpoint, the VKAs have been demonstrated to have a very narrow therapeutic window in patients with cirrhosis. The innate elevation in INR at baseline in the cirrhosis population makes drug monitoring challenging. Indeed, uncontrolled data have accumulated suggesting a higher than expected bleeding complication rate in cirrhosis patients placed on the VKA for PVT. ${ }^{[14]}$ Caution should be exercised in using these agents in the cirrhosis population. The use of LMWH in cirrhosis has been studied more extensively than any other class of agent and complication rates appear to be similar to the non-cirrhosis population. The inconvenience of multiple daily injections makes this therapy unappealing for long term use.The use of DOACs in this population has been studied in small case series in Child A and B patients and they appear as efficacious as LMWH with comparable safety profiles. ${ }^{[15,16]}$ Concerns over the reversal of the antihemostatic properties of the DOACs and, to a lesser extent, of the LMWHs, have been raised but the agents for quick reversal of actions of these drugs are in the final stages of development and approval in the U.S., Western Europe, and other countries. Case reports of successful reversal of dabigatran immediately before transplantation have been published. ${ }^{[17]}$

The notion of using TIPS accompanied by mechanical or therapeutic thrombolysis to reestablish flow in the portal vein is based on the longstanding practice in hepatic vein thrombosis. TIPS placement in the setting of active thrombosis can remove active clot and quickly reestablish physiologic flow in the portal vein in a matter of hours. Once again, randomized trials have not been completed, but in highly skilled hands, the success rates for recanalization of the portal vein either through a transhepatic approach ${ }^{[18]}$ or transplenic approach ${ }^{[19]}$ appear to be greater than $90 \%$. In addition to high recanalization rates, TIPS also improves portal hypertension globally, therefore reducing the risk of esophageal variceal bleeding and ascites formation. Disadvantages to this therapy include the need for highly specialized and experienced proceduralists able to accomplish the procedure, the permanent disruption of anatomy innate to TIPS placement, and procedural complications including worsening of hepatic encephalopathy. The literature is also unclear on the need for ongoing therapeutic anticoagulation after TIPS for this indication, especially in the setting of mechanical thrombolysis.

\section{CURRENT PRACTICE AND FUTURE DIRECTIONS}

Because of the scarcity of controlled clinical trial data in this area, practice guidelines are based on speculation, uncontrolled series, and expert opinion. Practice patterns vary widely based on local expertise. In the U.S., ready DOAC availability and general approval by most medical insurance companies have resulted in a predominantly medical approach as a first line therapy. Many centers with broad experience in the field of PVT and liver transplantation use a graded approach in patients eligible for PVT therapy:

If the patient has no major contraindication to TIPS and significant portal hypertension, especially high risk esophageal varices or poorly controlled ascites, TIPS can be used for these indications if it is technically feasible, based on the location and extent of the PVT. 
If mild portal hypertension conditions exist (but would not otherwise justify TIPS), medical therapy can be used for PVT.

Prior to initiation of medical therapy, patients who are not recently screened should undergo upper endoscopy to evaluate esophageal varices. Variceal bleeding risk should be controlled with effective beta blockade or eradication via endoscopic ligation prior to the initiation of anticoagulant therapy.

Medical therapy should be deferred until bleeding risk is assessed, but significant delays should be avoided. Therapy should be started within at least 6 months of diagnosis, but shorter delays are preferable where ever possible.

Center expertise and patient preference will determine the optimal medical agent and dose until specific dosing and pharmacokinetic data are available in the literature. Because of the above described difficulties in cirrhosis patients with the VKAs, most centers choose LMWH or the DOACs. Patient preference to avoid long term injections often ultimately results in DOAC use in the U.S.

Regular surveillance of patients with cross sectional imaging is used to assess treatment response approximately every three months.

The optimal length of therapy for antihemostatic drugs is not defined but given the relatively high recurrence rates after discontinuation of therapy, treatment until the time of transplantation or indefinitely may be considered.

If complete response to medical therapy is achieved, it is reasonable to lower medication doses to prophylaxis levels but further study is needed in this area.

If medical therapy is unsuccessful and there is progression or failure of response, TIPS can be used as a salvage therapy if no other contraindication and technically feasible. Optimal use of TIPS is most successful at experienced centers.

It is unknown whether medical anticoagulation should be used after TIPS for PVT as a maintenance therapy.

\section{CONCLUSION}

PVT is a recognized and common complication of cirrhosis. While its significance in the setting of the progression of liver disease is not clear, PVT is undoubtedly associated with significant morbidity and mortality, especially in the liver transplant candidate. Medical and revascularization therapies for PVT are available and can be used in the proper setting with good rates of success. Typically, in the U.S., medical therapy is attempted as a first line therapy if no contraindication and TIPS is used at specialty centers as a salvage therapy. Because clinical trial data is very sparse in this area, future controlled studies are needed in virtually all areas of this disease process to better define optimal timing, type, and duration of therapy.

\section{Funding Source}

None

\section{Conflict of Interests}

None declared.

\section{REFERENCES}

1. Francoz C, Belghiti J, Vilgrain V, Sommacale D, Paradis V, Condat B, et al. Splanchnic vein thrombosis in candidates for liver transplantation: usefulness of screening and anticoagulation. Gut 2005; 54:691-7.

2. Okuda K, Ohnishi K, Kimura K, Matsutani S, Sumida M, Goto N, et al. Incidence of portal vein thrombosis in liver cirrhosis. An angiographic study in 708 patients. Gastroenterology 1985; 89:279-86.

3. Yerdel MA, Gunson B, Mirza D, Karayalcin K, Olliff S, Buckels J, et al. Portal vein thrombosis in adults undergoing liver transplantation: risk factors, screening, management, and outcome. Transplantation 2000; 69:1873-1881.

4. Sarin SK, Philips CA, Kamath PS, Choudhury A, Maruyama H, Nery FG, et al. Toward a Comprehensive New Classification of Portal Vein Thrombosis in Patients With Cirrhosis. Gastroenterology 2016; 151:5747, e573.

5. Englesbe MJ, Kubus J, Muhammad W, Sonnenday CJ, Welling T, Punch JD, et al. Portal vein thrombosis and survival in patients with cirrhosis. Liver Transpl 2010; 16:83-90.

6. Stine JG, Pelletier SJ, Schmitt TM, Porte RJ, Northup PG. Pre-transplant portal vein thrombosis is an independent risk factor for graft loss due to hepatic artery thrombosis in liver transplant recipients. HPB (Oxford) 2016; 18:279-86.

7. Villa E, Camma C, Marietta M, Luongo M, Critelli R, Colopi S, et al. Enoxaparin prevents portal vein thrombosis and liver decompensation in patients with advanced cirrhosis. Gastroenterology 2012; 143:1253-60, e1251-4.

8. Nery F, Chevret S, Condat B, de Raucourt E, Boudaoud L, Rautou PE, et al. Causes and consequences of portal vein thrombosis in 1,243 patients with cirrhosis: results of a longitudinal study. Hepatology 2015; 61:660667.

9. Garcia-Tsao G, Abraldes JG, Berzigotti A, Bosch J. Portal hypertensive bleeding in cirrhosis: Risk stratification, diagnosis, and management: 2016 practice guidance by the American Association for the study of liver diseases. Hepatology 2017; 65:310-35.

10. DeLeve LD, Valla DC, Garcia-Tsao G, American Association for the Study Liver D. Vascular disorders of the liver. Hepatology 2009; 49:1729-64.

11. Qi X, De Stefano V, Li H, Dai J, Guo X, Fan D. Anticoagulation for the treatment of portal vein thrombosis in liver cirrhosis: a systematic review and meta-analysis of observational studies. Eur J Intern Med 2015; 26:23-9.

12. Hibi T, Nishida S, Levi DM, Selvaggi G, Tekin A, Fan J, et al. When and why portal vein thrombosis matters in liver transplantation: a critical audit of 174 cases. Ann Surg 2014; 259:760-6. 
13. Tripodi A, Mannucci PM. The coagulopathy of chronic liver disease. $\mathrm{N}$ Engl J Med 2011; 365:147-56.

14. La Mura V, Braham S, Tosetti G, Branchi F, Bitto N, Moia M, et al. Harmful and Beneficial Effects of Anti-coagulants in Patients With Cirrhosis and Portal Vein Thrombosis. Clin Gastroenterol Hepatol 2017; October 21. [Epub ahead of print]

15. Intagliata NM, Henry ZH, Maitland $\mathrm{H}$, Shah NL, Argo CK, Northup PG, et al. Direct Oral Anticoagulants in Cirrhosis Patients Pose Similar Risks of Bleeding When Compared to Traditional Anticoagulation. Dig Dis Sci 2016; 61:1721-7.

16. De Gottardi A, Trebicka J, Klinger C, Plessier A, Seijo S, Terziroli B, et al. Antithrombotic treatment with direct-acting oral anticoagulants in patients with splanchnic vein thrombosis and cirrhosis. Liver Int 2017; 37:694-9.
17. Intagliata NM, Maitland H, Pellitier S, Caldwell SH. Reversal of direct oral anticoagulants for liver transplantation in cirrhosis: A step forward. Liver Transpl 2017; 23:396-7.

18. Wang L, He F, Yue Z, Zhao H, Fan Z, Zhao M, et al. Techniques and long-term effects of transjugular intrahepatic portosystemic shunt on liver cirrhosis-related thrombotic total occlusion of main portal vein. Sci Rep 2017; 7:10868.

19. Thornburg B, Desai K, Hickey R, Kulik L, Ganger D, Baker T, et al. Portal Vein Recanalization and Transjugular Intrahepatic Portosystemic Shunt Creation for Chronic Portal Vein Thrombosis: Technical Considerations. Tech Vasc Interv Radiol 2016; 19:52-60.

How to cite this article: Northup PG, Davis JPE. Timing of anticoagulation for portal vein thrombosis in liver cirrhosis: A US hepatologist's perspective. J Transl Intern Med 2018; 6: 1-5. 